\title{
“ESTRATEGIAS EDUCATIVAS PARA EL USO DE LAS NUEVAS TECNOLOGIAS DE LA INFORMACION Y COMUNICACION"
}

\author{
MSc. BÁRBARA LABORÍ DE LA NUEZ \\ Instituto Superior Politécnico "José Antonio Echeverría”, La Habana \\ Dr. IÑIGO OLEAGORDIA AGUIRRE \\ Universidad del País Vasco
}

\section{INTRODUCCIÓN}

El presente trabajo surge en primer lugar de la necesidad de adaptar a las circunstancias actuales el proceso formativo de los titulados de la Escuela de Ingeniería Técnica de la Universidad del País Vasco (UPV). Desde hace tiempo se viene hablando sobre el papel y las oportunidades que las nuevas tecnologías ofrecen en todos los campos sociales y en especial en la formación. Sin embargo, salvo excepciones, la aplicación sistemática de la tecnología a la formación ha ido siempre bastante retrasada respecto a las posibilidades que en cada momento se ofrecían. Si actualmente las esperanzas de implantación generalizada pueden convertirse en realidad, fundamentalmente es como consecuencia de la concurrencia de dos factores: la tecnología multimedia y el nuevo modelo de sociedad.

De hecho desde hace tiempo existe tecnología como para posibilitar otras modalidades de formación dentro y fuera del ámbito universitario. Una posible explicación a este hecho aparentemente contradictorio, es que la formación tiene unas connotaciones sociales que sobrepasan los aspectos técnicos para modificar las relaciones humanas. Es en este punto donde la nueva sociedad que se está formando tiene mucho que decir sobre el cambio de las costumbres y formas de entender el papel de las nuevas tecnologías.

La actividad educativa es una de las más complejas del ser humano. Es por ello que se ha investigado y se investiga la operatividad de distintos modelos educativos, dentro de los paradigmas imperantes del momento, empleando distintos recursos didácticos que produzcan aprendizaje significativo y activo del estudiante.

El diseño de aplicaciones multimedias presupone la conjunción de dos partes inseparables: la pedagogía y la tecnología. De la pedagogía se tiene en cuenta la selección de los métodos educativos empleados para lograr la participación del estudiante como un ente activo y la utilización de la tecnología implica la utilización y combinación de las modalidades de la informática educativa para lograr un producto informático que cumpla con los objetivos propuestos.

En las secciones siguientes se exponen las características y selección del modelo educativo y de la modalidad de la informática que se considera más conveniente utilizar dadas las características de los contenidos de las asignaturas electrónicas, conduciendo a la adopción de nuevos métodos donde la enseñanza presencial pierde su hegemonía actual y pasa a ser sustituída por sistemas en los que la información se comunica al alumno de forma diferente a la tradicional.

\section{MODELOS EDUCATIVOS}

En un ambiente universitario donde los destinatarios y principales usuarios de la aplicación informática van a ser fundamentalmente los alumnos, es necesario un análisis de los modelos 
educativos más representativos œn el objetivo de que sirva de pauta para el diseño de aplicaciones informáticas de carácter didáctico.

Se describen por separado los aspectos más significativos de dos metodologías: conductista y cognitiva, para su utilización, en función de la materia de estudio, preferencias educativas y dinámica de aprendizaje. Se trata de comprobar si es posible crear los estímulos que activen y aceleren el aprendizaje, mediante un clima adecuado, que provoque la actividad del alumno, le motive adecuadamente y permita después al profesor dedicarse a profundizar en las incógnitas que el recurso utilizado le está suscitando. En este sentido es preciso pensar, fundamentar y desarrollar un determinado modo de relación entre las NTIC y la educación que bien podríamos calificar de integrador que permita al estudiante ocupar un papel más activo en el proceso de aprendizaje.

\section{a. MODELOS COGNITIVOS}

La palabra cognición implica un área vagamente definida que abarca todo el complicado sistema de las capacidades mentales del ser humano, centrándose en la percepción, atención, aprendizaje y memoria; en la comunicación y comprensión del conocimiento así como en el razonamiento. Tradicionalmente y de una forma general se concibe la cognición como el conocimiento por la inteligencia. En este sentido la utilización del conocimiento en el razonamiento y en la solución de problemas es un tema central de análisis y estudio en la cognición.

En el desarrollo de la aplicación informática el objetivo principal de este tipo de modelos es la interactividad, el desarrollo cognoscitivo y la capacidad intelectual en general. De esta forma se consigue estimular, fomentar y potenciar por parte de los alumnos la capacidad de procesar la información comunicada de forma que mejore la adquisición de conocimientos y la aplicación de los mismos.

Los modelos más representativos de este tipo son:

1. Modelo de organización intelectual ${ }^{1}$

Este modelo se centra en potenciar la eficacia para procesar adecuadamente la información comunicada. La metodología consiste en integrar los aprendizajes parciales en estructuras más ricas en contenido y por lo tanto más complejas. Este proceso se dirige mediante la asimilación de conceptos fundamentales que, como "andamiajes intelectuales" estructuran de una forma ordenada ideas y hechos. La operatividad de este modelo se consigue relacionando distintos aspectos dentro de la propia unidad de conocimiento.

\section{Modelo de desarrollo cognoscitivo}

El modelo está concebido para potenciar el desarrollo intelectual general y el lógico en particular. Se aplica fundamentalmente en entornos informáticos ${ }^{7}$.

\section{Modelo de formación de conceptos y descubrimiento ${ }^{2}$}

El objetivo es desarrollar el pensamiento inductivo y el análisis de conceptos. La inducción permite la obtención de leyes de validez universal mediante el análisis de un número reducido de casos. Se trata de un método eminentemente pragmático, y de difícil aceptación por aquellos que no están familiarizados con él, por proceder a una generalización a partir de situaciones puntuales.

Sin embargo hay situaciones en las que proceder a un estudio riguroso de un fenómeno, puede exigir un esfuerzo que no compense a la información que proporciona. 


\section{Modelo de pensamiento inductivo e investigación ${ }^{8}$}

$\mathrm{Su}$ finalidad estriba en desarrollar los procesos mentales inductivos y deductivos, el razonamiento académico, el inicio, desarrollo e implementación de teorías. Mediante la deducción se parte de ciertos principios generales, que son evidentes por sí mismos o de intuición inmediata, y con la ayuda de la azón se deduce todas las consecuencias que de ellos se derivan. Este modelo se basa frecuentemente en la elaboración de teorías, previo establecimiento de hipótesis de trabajo y experimentación o simulación de los hechos que suceden en el mundo real.

\section{b. MODELOS CONDUCTISTAS}

Los modelos conductistas, ampliamente empleados en la enseñanza, están basados en un cuerpo doctrinal llamado conductismo que tiene como objetivo fundamental la evolución del usuario en lo que a conducta operativa se refiere. Están basados fundamentalmente en el principio de una emisión controlada de estímulos de diversa naturaleza. Cuando se trata de evaluar el grado de conocimiento adquirido se efectúa un análisis posterior de las respuestas emitidas. Dicho modelo ha sido y sigue siendo utilizado en numerosos medios educativos e instructivos.

Se expondrá a continuación las características de algunos modelos conductistas.

\section{Modelo de entrenamiento directo}

Este modelo se ha desarrollado sobre la psicología del entrenamiento, que acentúa el análisis de tareas y el diseño de los componentes del entrenamiento, la teoría de sistemas que se ocupa del diseño de sistemas, entendiendo que todo comportamiento humano opera como parte de un sistema de organización dentro de un entorno determinado. En este sentido se debe presentar especial atención a los problemas derivados del autocontrol y realimentación donde la persona centra su interés mediante la realimentación sensorial para controlar y modificar su propia estrategia de aprendizaje. El conductismo o la psicología conductista que pone el acento en las técnicas de modelado y analogías como método de asimilar y adquirir nuevos conocimientos. La analogía se basa en las similitudes que presentan los fenómenos objeto de estudio con otros ya conocidos, como es el caso del estudio de sistemas distintos pero caracterizados por modelos matemáticos muy parecidos o iguales.

\section{Modelo del refuerzo y del autocontrol}

Este modelo está fundamentado en el control de estímulos y acciones a realizar, así como en el refuerzo positivo, que controlados por el propio usuario sobre sí y sobre el medio desarrolla la autoestima y la confianza de asimilar y desarrollar el conocimiento presentado. Basado el modelo en la concepción de la conducta humana como función del ambiente inmediato, su carácter esencial es la relación que existe entre el estímulo y la respuesta. El llamado refuerzo contingente que aplica el control sistemático de estímulos reforzantes que se presentan cuando se produce una deseada respuesta.

\section{INFORMÁTICA EDUCATIVA}

La enseñanza, utilizando las nuevas tecnologías hoy disponibles, debe dar una información que proporcione una visión global de los conceptos fundamentales y que permita prever el resultado u objetivo final, el control de los comportamientos, la formulación de un programa, su aplicación y evaluación consiguiente. Debe permitir añadir ejemplos de aplicaciones reales de las ideas 
expuestas, para convertir los conceptos en algo vivo para el estudiante y la existencia de una unidad de simulación que permita al educando plantear sus propios casos y resolverlos, siguiendo el sistema de ver ¿qué pasaría si...... ?. Las recientes teorías del aprendizaje propugnan que el conocimiento es algo que cada individuo reconstruye, y por lo tanto el conocimiento no se adquiere por mera transmisión. En consecuencia se sostiene que las estrategias del aprendizaje más efectivas son las que explotan el principio de aprender haciendo.

\section{a. Instrucción programada}

La enseñanza programada es una aplicación directa cuya finalidad es la de provocar estímulos sistemáticos y refuerzos inmediatos y es una de las aplicaciones del modelo conductista en el ámbito educativo. Si los programas pretenden ante todo proporcionar oportunidades de realizar ejercicios relativos a contenidos o temas que ya le han sido introducidos, se tiene otra modalidad de instrucción programada denominada ejercitación. En este caso el ordenador puede proporcionar las explicaciones teóricas pertinentes sobre todo si va combinada con aplicaciones tutoriales, o comunicar el alumno con el profesor o con otras fuentes de información o consulta. Los efectos educativos de la interactividad se acrecientan al registrar los datos que genera el estudiante en su labor y al usarlos como parámetros en el proceso de aprendizaje. Para que la ejercitación pueda tener un valor pedagógico se precisan programas didácticos y escritos con un uso adecuado de los recursos interactivos.

\section{b. Tutorial}

Un ordenador que ejecuta un programa de tipo tutorial instruye a un estudiante en una determinada rama de conocimientos de forma análoga, en cierto modo, a la que emplearía un profesor en situación de enseñar a un sólo alumno. Evidentemente, hay diferencias esenciales. El ordenador no es un ser humano; es solamente una máquina programada para transmitir unos conocimientos de forma semejante a un profesor pero severamente restringida. Los tutoriales más perfeccionados llegan a admitir, hasta un cierto punto, respuestas constructivas y pueden utilizar una amplia gama de recursos (gráficos de alta resolución en color, sonido, pantalla táctil, etc. ). Como inconvenientes se acepta que la escasa inteligencia y flexibilidad de los programas de ordenador obliga a trivializar los diálogos tutoriales a través de un estrecho marco de opciones, que es insuficiente para presentar y explorar convenientemente conceptos complicados. Aceptando estas limitaciones, de por sí importantes, en sentido contrario se puede argumentar que este defecto puede restringir la operatividad del mismo si el ordenador no dispone de una base de información implementada en forma de hipertexto o constituye el único medio de instrucción. La instrucción programada es una raíz común de la ejercitación y el tutorial, por lo que ambos tipos de aplicaciones forman a menudo un todo único por lo que fueron las primeras aplicaciones de los ordenadores a la enseñanza dando lugar a la denominada "instrucción asistida por ordenador" (C. A. I.) "Computer Assisted Instruction".

\section{c. Simulación}

Se trata de programas capaces de simular en el ordenador situaciones reales y generar resultados precisos. Son muy interesantes puesto que la realización expresa de programas "ad hoc", permite abordar la resolución de problemas cuya finalidad, cuando no se dispone de dichas herramientas, obliga a tratarlos de forma teorizante. En general el modelado y la simulación surgen de forma natural como extensión del concepto de diseño. El diseño supone la manipulación de 
elementos que representan posibilidades reales, es decir se basa en modelos creíbles de la realidad. Un diseño cuidadoso impulsa a la investigación de la naturaleza y construcción del modelo, entendidos éstos como una representación compacta del fenómeno real.

La aparición del ordenador cambió la situación de forma radical al introducir una herramienta para la representación simbólica de los modelos y el cálculo numérico de su comportamiento. En este contexto, un modelo de simulación se puede considerar como un conjunto de ecuaciones para generar el comportamiento del sistema real. El ordenador, bajo el control de un programa que implementa el modelo, puede emplearse para generar su comportamiento. Este proceso se denomina simulación y el programa, programa de simulación.

En las simulaciones el acento recae en la interacción individualizada del usuario con el modelo a través del programa, para lo cual se sumerge en el mundo creado por el modelo. Así pues, el verdadero poder de las simulaciones radica en el desarrollo e incentivación del pensamiento y de la intuición, en la invención y contraste de hipótesis, y también en la posibilidad que brindan de comprender lo esencial de ciertas situaciones, actuando de la forma más coherente, en lugar de preocuparse del aprendizaje de contenidos.

La simulación constituye una de las aplicaciones más fructíferas e imaginativas de los ordenadores en la enseñanza. Dos son sus aportaciones fundamentales: en primer lugar, la simulación encaja plenamente en los objetivos normales de los planes de estudio, complementando los restantes procedimientos didácticos, pudiendo llegar a ser tan formativa e interesante como el trabajo de laboratorio. En segundo lugar, la relativa simplicidad de los algoritmos de los modelos que se emplean, permite que los propios profesores puedan plasmarlos en programas de actualidad tecnológica que se ajustan a las prestaciones usuales de los ordenadores presentes en las instituciones educativas.

Otra concepción de la simulación que entronca con la utilización del ordenador como instrumento de control, se obtiene cuando la entrada y salida de datos se dirige desde o hacia dispositivos físicos, y se reserva el monitor y el teclado para la dirección y control del experimento. En este caso la simulación se realiza con instrumental de laboratorio y el ordenador es el intermediario entre dicho instrumental y el propio experimentador.

\section{d. Niveles multimedia}

Cuando se habla del empleo de los "multimedia", se está haciendo referencia a la utilización a través del ordenador de 'múltiples medios' como texto, gráficos, sonido, imágenes, animación y simulación, que son combinados y controlados por el usuario de forma interactiva, para conseguir un efecto deseado.

El uso de color, imagen, sonido y animación no es excesivamente reciente, pero sí lo es su empleo en la elaboración de cursos, con las técnicas 'multimedia'. La razón es simple: el gran espacio ocupado en memoria cuando se almacenan ficheros de sonido o imagen, digitalizados, hacía inviable su uso con ordenadores normales. La aparición de los nuevos sistemas de almacenamiento mayoritariamente CD-ROM, ha hecho factible su empleo, utilizando interfaces y periféricos adecuados.

Por otro lado, los grandes avances obtenidos recientemente en el mundo de las comunicaciones han hecho posible la transmisión de cursos mediante redes locales, redes telefónicas, radio, fibra óptica, vía satélite, etc., con lo que la educación puede hacerse llegar con 
facilidad hasta el lugar deseado. Esto ha abierto nuevas y amplias perspectivas en la educación a distancia.

\section{e. Hipertexto y multimedia: hipermedia}

Hasta ahora la enseñanza siempre se ha desarrollado de forma casi lineal. Esto quiere decir que las unidades en las que puede dividirse el conocimiento se transmiten de forma consecutiva, es decir, se informa y comunica una después de la otra. El problema está en que el proceso de aprendizaje de la persona no siempre es lineal. La mente humana asimila, tanto de una forma profunda mediante el estudio, como de una forma horizontal a través de la experiencia. Adquiere las distintas unidades de conocimiento y as asocia, en profundidad o en paralelo, siguiendo un camino diferente, elegido personalmente. El hipertexto es un sistema que permite comunicar la información, dividiéndola en unidades (nodos), ligados de forma tal, que el usuario puede acceder a la información que él personalmente decida en cada momento. Este sistema de ir de un punto a otro se denomina navegación. Por lo tanto el hipertexto permite establecer una representación prácticamente no lineal del conocimiento a través de una serie de unidades básicas del mismo (nodos) situadas a distintos niveles y ligadas entre sí por medio de relaciones jerárquicas y asociativas. Las uniones entre nodos son establecidas por el profesor o por el mismo sistema en función de la experiencia (Sistema Inteligente), permitiendo "navegar" al alumno entre ellos a su propia voluntad.

La posibilidad de utilizar en los nodos texto, gráficos, simulaciones, imágen y sonido, permite llegar a una representación del conocimiento, de una manera atrayente y motivante, mediante la combinación del conocimiento profundo y del horizontal (experiencia), permitiendo su comunicación al alumno de una forma eficiente, consistente y oportunista, dando lugar a una correcta asimilación y acomodación del mismo, es decir, a un aprendizaje ${ }^{4}$. Un hipertexto, combinado con los multimedia, ofrece lo que llama Nielsen ${ }^{6}$ los hipermedia: herramienta óptima para la transmisión de conocimientos en el entorno de la educación continua, flexible, abierta y a distancia.

\section{EVOLUCION DE LAS NECESIDADES EDUCATIVAS}

En el ámbito universitario, la educación tradicional se ha centrado, durante un largo período de tiempo, en una enseñanza académica, quizás demasiado teórica, que debido a las mutaciones producidas en la sociedad con la aceleración de los cambios actuales, ha requerido orientarse hacia unos nuevos planes de estudio más cortos, menos academicistas y más prácticos ${ }^{3}$.

La aceleración del cambio tecnológico hace cada vez más caduco el estático planteamiento educativo tradicional. En particular, la práctica inexistencia de formación continua de los profesores constituye un claro ejemplo del escaso valor de mercado que la sociedad generalmente asigna a la enseñanza. La sociedad siente la inadecuación de los productos que proporcionan los sistemas educativos formales con relación a las necesidades del presente y del futuro inmediato. Producto de ello es el deseo, a menudo no explícito, de una revisión a fondo de las estructuras modificándolas de forma tal que permitan una mayor y más rápida adaptación. El cesarrollo de nuevas industrias y tecnologías y el declive de viejos procesos llevan a la aparición de nuevas profesiones y nuevos modos de producción y gestión, por ende, se sugiere la necesidad de una educación permanente y continuada. 
Los problemas que se intuyen en el futuro cercano radican, en buena medida, en la incapacidad de los sistemas educativos para anticiparse y acomodarse a los cambios sociales, los cuales hoy en día están condicionados, entre otros factores, por el cambio tecnológico. En este sentido, en educación el cambio se considera como la respuesta a un problema identificado.

Es evidente que los sistemas educativos del futuro deberán ser de una capacidad de adaptación muy superior a la actual. La formación cíclica, continua y diversificada será una de las fórmulas a adoptar. La promoción de nuevos productos y servicios de la tecnología de la información está ya ejerciendo un fuerte tirón en la demanda de personal capacitado para operar, gestionar, diseñar, construir y explotar los diversos elementos constituyentes de los sistemas de información y comunicación. En general, la educación actual aún no está preparada para asumir y conducir este cambio de rumbo. Así mismo, es preciso ir preparando una fuerza laboral e intelectual muy creativa y cualificada para abordar los problemas que, por la rápida evolución de la tecnología, no se podrán resolver a medio plazo si no se toman las medidas a tiempo.

Todo ello supone una reducción en la eficacia total del equipo humano, si no se recurre a la actualización y adecuación de sus conocimientos, para que pueda mantenerlos al día y satisfacer las nuevas necesidades. En una palabra, se precisa el reentrenamiento de la fuerza intelectual existente $^{5}$. En la formación universitaria se han seguido métodos dásicos de educación presencial, excepto en la UNED ( España), quedando sin posibilidades de acceder a ella todos los colectivos en los que ha sido prácticamente imposible, - por razones geográficas o económicas, - la implantación de la enseñanza tradicional.

Dentro de los planes de estudio, durante los años de formación, la enseñanza clásica ha sido, en general, monolítica. Es decir: el desarrollo curricular ha sido fijo y estable, orientado a la adquisición de unos conocimientos teóricos, más que al logro de un cierto grado de aplicación de los conocimientos adquiridos siguiendo un modelo educativo totalmente conductista carente de la más mínima flexibilidad.

La enseñanza ha estado basada, fundamentalmente, en la lección magistral impartida por el profesor en el aula, donde el alumno podía hacer las preguntas deseadas y tomar apuntes de lo dicho. Luego, con esos apuntes y los libros de texto y consulta convencionales, - en una palabra con información "impresa", - procedía al estudio individual. La realización de ejercicios, problemas y casos, de forma aislada o en el mismo aula, junto con la realización de prácticas de forma masificada o en número insuficiente, permitía ver la aplicación de esa enseñanza a la práctica y servía para reforzar los conceptos adquiridos.

La tecnología de la comunicación está perfectamente enraizada con la información dando lugar al desarrollo de las Tecnologías de la Información y Comunicación (TIC). La cultura colectiva está cambiando esencialmente de estar soportada por los libros a estar basada en el ordenador a través de los medios y soportes de información y comunicación que conforman las TIC. Hasta ahora, y por diversas razones, el efecto que se ha producido en la educación reglada (formal) ha sido más amortiguada que en otros tipos de formación. Respecto a los niveles de enseñanza formal se ha de decir que el universitario, que desde los primeros tiempos ya estuvo presente en la encrucijada de las nuevas tecnologías de la información y en posesión de sensibles recursos económicos y de innovación, ha malgastado, en general, largos años de inacción. 
La falta de técnicas pedagógicas y la complejidad de conocimientos, surgida sobre todo con la aparición de las TIC en las áreas de conocimiento científico y técnico, hace muy difícil seguir con este sistema. Es necesario la conformación de los tipos de tecnología para la enseñanza superior, la investigación y la educación continua de ámbito profesional, que se precisará para el horizonte de este siglo.

Hay que implementar proyectos avanzados que tengan como objetivo fundamental ayudar a los profesores y centros para preparar a los alumnos para la vida en una sociedad en la que los dispositivos y los sistemas basados en la microelectrónica serán de utilización general. A tal efecto se debe dirigir la investigación de las formas más apropiadas de usar el ordenador como instrumento de enseñanza, investigación y de aprendizaje en situaciones diversas y la introducción de nuevos temas en los planes de estudio, en forma de nuevas materias y de aportaciones originales a los temas habituales de enseñanza.

Dentro de un programa de "alfabetización informática" nos encontramos, por lo tanto, enfrentados a la urgente necesidad de investigar qué es lo que hay de básico en el empleo del ordenador. Por todo ello, deben investigarse todas las formas y medios de utilizar los ordenadores para activar la educación haciendo un esfuerzo intelectual y organizativo para que este proceso imparable adopte una forma educativa profunda y viable. La segunda alfabetización se producirá cuando la educación permita convertirlos en herramientas intelectuales de amplio uso y en interlocutores valiosos en muchas esferas de la actividad humana.

Las TIC son herramientas potentes en cuanto a la posible información que orecen y con relación a la capacidad de acción y de actuación que ejercen sobre los usuarios. La técnica presenta la posibilidad de la interactividad como fuente comunicativa entre individuos, objetos, conocimiento y maneras de procesar la información. Hay que señalar que un gran número de tecnologías ya se viene incorporando a actividades comunes, en el hogar y en el trabajo. En definitiva son el fruto de una sociedad industrial, de la información y del conocimiento generado por ella que está suponiendo una profunda transformación en múltiples campos de la actividad humana.

Las TIC aportan medios poderosos que deben ponerse al alcance de todos los individuos y además deben mantenerse en contacto e interacción con la realidad, ya que en la actualidad la vida académica y de formación presencial exclusivamente en la Universidad se va restringiendo día a día. Un porcentaje considerable de oferta educativa de postgrado se concentra, en la actualidad, en empresas dedicadas a formación.

Aún cuando las TIC tienen gran velocidad de penetración y poder de cambio con relación a los procesos de trabajo, se observa cómo existe una limitación del uso, o de la incorporación de las mismas en los sectores educativos tradicionales, alejados de lo que viene pasando en la industria y en empresas privadas. Esto puede potenciar la aparición de un uso de la tecnología educativa con idea de entrenamiento, orientada a la adquisición de habilidades de operación y destrezas en detrimento de adquisición de conocimientos que superen la repetición o la mecánica. Este estilo de enseñanza, puede crear un rechazo en los medios académicos, cuya colaboración es sin embargo imprescindible para una creación de calidad puesto que el trabajo de investigación es imprescindible. 


\section{LAS T.I.C Y LA ENSEÑANZA}

Hablar de las TIC en la enseñanza, en el aprendizaje, en la formación, exige ubicarse en el tema relativo a los "Medios y Recursos" que incorporamos para desarrollar actividades, contenidos y objetivos educativos. De ahí que sea fundamental elegir y considerar el tipo de medio que vamos a emplear, para aseguramos de la actividad mental que estimula, la facilidad que tenga para transmitir información, la capacidad para conectar con las características cognitivas de los alumnos y situarnos en un punto de partida eficaz. Por lo tanto al optar por las TIC, como soportes y canales para el tratamiento y acceso a la información, se haga entendiéndolas como nuevas herramientas y nuevos modos de expresión que suponen nuevas formas de acceso y nuevos modelos de participación.

Una de las finalidades de la educación es capacitar al alumnado para comprender, crear y participar en la cultura de su tiempo. Las TIC suponen en ese ámbito una nueva forma de organizar, representar y codificar la realidad, son además instrumentos valiosos para lograr un elevado grado de aplicación de los conocimientos adquiridos. El profesional de la enseñanza no puede permanecer ajeno a esta situación, por lo que debe hacer un esfuerzo en lo que a la actualización tecnológica se refiere una vez superada la posible intimidación que la tecnología puede suponer. Las tecnologías del aprendizaje representan una de las fuerzas renovadoras en los sistemas de aprendizaje y constituyen un elemento clave para el desarrollo de la educación y la formación.

Consecuentemente la investigación sobre la mejor manera de fortalecer la tecnología para propósitos de aprendizaje, tendrá una fuerte influencia en la construcción de las futuras formas de aprendizaje. En este sentido es preciso pensar, fundamentar y desarrollar un determinado modo de relación entre las nuevas tecnologías y la educación que se debe calificar de integrador.

\section{EL SISTEMA EDUCATIVO.}

La educación se puede considerar como un sistema que presenta una dualidad notoria: por un lado es abierto e interactúa con un entorno social, político, cultural y económico, por otro se comporta como un sistema cerrado de bajo dinamismo, ya que dispone de elementos internos capaces de lograr sus objetivos y establecer esquemas de medición, evaluación, y corrección de sus resultados. Como sistema presenta elementos, flujos de información, entradas de recursos de transformación (humanos, financieros, de conocimiento) y salidas.

Es en la vertiente de sistema cerrado donde deseamos analizar algunos aspectos del papel de las técnicas informáticas derivadas de las TIC, considerando la educación como un sistema formalizable que posee una estructura organizativa y que, mediante la utilización de diversos recursos aplicados según una colección de criterios y normativas, consigue los objetivos que se le adscriben con un grado controlable de discrepancia. En los apartados anteriores se ha insistido en la necesidad de cambiar el enfoque de la educación; en éste se enfatiza la urgencia de replantear la gestión. Actualmente, la importancia de la tecnología no se refleja adecuadamente en las prioridades educativas.

A continuación se sugieren varios puntos de reflexión en la planificación de un sistema educativo: 
1. Investigación y desarrollo, para analizar y definir las necesidades de innovación al objeto de orientar una política de investigación de nuevos métodos y sistemas, más aplicadas que académicas.

2. Eficacia financiera, en el sentido de diversificación de fuentes de financiación.

3. Sistematización de la planificación: significa elaborar métodos que permitan enlazar el sistema educativo con la planificación de las restantes parcelas vitales del sistema socioeconómico y la política de desarrollo científico.

4. Cooperación internacional para fomentar el intercambio de información, experiencias y soluciones innovadoras, refuerzo de los recursos nacionales y eliminación de trabas y discriminaciones.

Algunas acciones progresivas que podrían concertarse en aras a la construcción de un sistema de información educacional integrado, utilizable por gran número de instituciones o centros docentes, son:

1. Acuerdos de cooperación interinstitucional en el diseño, implantación y mantenimiento de bases y bancos de datos educacionales.

2. Establecimiento de funciones y comités de asesoramiento en cuestiones de estandarización.

3. Experimentación de núcleos de trabajo en gestión que operen con mentalidad de red ("networking").

La aplicación de las TIC complicará las cosas y defraudará las expectativas si no va acompañada de una organización adecuada. Se deben cambiar tanto las actitudes como los métodos. Estos dos últimos elementos deben darse en el personal implicado para que se produzcan condiciones favorables que potencien la detección de factores restrictivos, la consolidación de juicios propios, la capacidad de anticiparse y prever con eficacia, y la mejora de la actitud para el razonamiento lógico. Solamente si se dan estas circunstancias vale la pena continuar en el intento.

\section{NUEVAS NECESIDADES EDUCACIONALES Y NUEVO ENFOQUE PEDAGOGICO}

A la vista de lo expuesto, se precisa, cada día con mayor urgencia, abordar con nuevos métodos, dentro de las TIC, la enseñanza en tres vertientes a la vez: la fundamental, la enseñanza presencial y la educación continua a lo largo de toda la vida profesional de la persona. Se plantean, pues, una serie de necesidades actuales, que pueden sintetizarse en los siguientes puntos:

1. Es necesario proporcionar una formación en aspectos fundamentales y que además sea operativa.

2. La obsolescencia y el olvido, suponen una reducción en la eficacia total del conocimiento humano, si no se recurre a la actualización y adecuación de sus conocimientos, para que pueda mantenerlos al día y satisfacer las nuevas necesidades tecnológicas mediante el adecuado reentrenamiento.

3. Se precisa una adecuada actualización de los conocimientos de los grupos que acceden de nuevo al mundo laboral que en un momento abandonaron (reincorporación).

4. Es preciso reconducir la formación de aquellas personas cuyo puesto de trabajo actual se vea sometido a una reconversión (readaptación). Este fenómeno, debido fundamentalmente al 
ritmo de cambio tecnológico, requiere una inversión masiva en actualizar el nivel de conocimiento del personal técnico.

5. Hay que crear un efecto sinérgico, entre educación, entrenamiento y vida económica, que asegure la óptima aplicación de conocimientos adquiridos.

Es preciso, pues, desarrollar nuevas técnicas educativas de carácter integrador y ofrecer nuevas oportunidades. La nueva educación universitaria, - tanto básica, como continua, - no puede estar acotada por los cánones más o menos rígidos que han caracterizado a la educación clásica.

Para satisfacer todo esto es necesario que la persona pueda:

1. Primero y primordialmente adquirir una educación universitaria básica adaptada a la realidad de los nuevos cambios tecnológicos.

2. Poder mantenerse al día, compensando la obsolescencia y el olvido y adquirir así los conocimientos precisos para acceder con éxito al mercado laboral o a nuevas posiciones en la empresa u organización donde presta sus servicios.

3. Tener alternativas a la enseñanza reglada y presencial, que le permitan satisfacer sus aspiraciones en el entorno de estudio más adecuado.

4. Aprovechar su trabajo personal para mejorar su especialización: aprender haciendo.

5. Poder diseñar su propio curriculum personal, adecuado a sus características, posibilidades y necesidades.

6. Disponer de opciones de formación flexibles, que le permitan adaptarla a sus posibilidades en tiempo, espacio y ritmo.

\section{La educación universitaria bási ca y continua.}

Como enfoque para cubrir estas necesidades es necesario desarrollar un entorno educativo donde las personas involucradas tengan facilidades para que:

1. Puedan adquirir una educación básica universitaria adaptada a la realidad de las nuevas situaciones.

2. Tengan acceso a una educación continua, puesto que continuo es el deterioro de los conocimientos por obsolescencia y olvido y continua, también, la necesidad de llevarlos a un nivel superior, a lo largo de la vida profesional.

Se puede decir, esumido en una sola frase, que "aprender debe ser un proceso continuo de la vida y del trabajo diario ".

En concreto, para la educación continua, se precisa:

1. Establecer un sistema de actualización de conocimientos en el tiempo, que permita a los graduados de hace 3 a 5 años, poner al día su saber, es decir, adecuarlo al que tiene un estudiante que se gradúa en la actualidad.

2. Fijar un camino para la adecuación del nivel de conocimientos de la fuerza de trabajo a los nuevos puestos que van ocupando en su carrera profesional.

3. Dar una formación a medida del usuario, que es la solución que van buscando, cada vez más, las empresas, los sectores industriales y los propios individuos aislados.

La educación continua proporciona una adecuación de la competencia profesional en tiempo real: 
1. Facilitando el aprendizaje de las nuevas tecnologías y el empleo de nuevas aplicaciones.

2. Desarrollando una especialización como parte integral de la competencia profesional esencial para todas las ramas de la ingeniería.

3. Ejercitando el desarrollo mental continuo, para alcanzar objetivos nuevos y más elevados.

4. Por otro lado, estimular un aprendizaje activo, en vez de la formación de la enseñanza clásica:

- A base de un programa individualizado en vez de las 'clases'.

- Desarrollo de trabajo en lugar de 'cursos'.

- Relación permanente en vez de esporádica.

- Aprendizaje a partir de información electrónica en vez de libros.

- Solución de problemas y toma de decisiones en vez de memorizar información.

- Aprendizaje basado en las necesidades reales en vez de en objetivos de aprendizaje.

\section{Educación flexible abierta y a distancia}

Hay que tener en cuenta la posibilidad de que esta formación vaya a ser dirigida a personas que no pueden recibir una educación presencial y sólo pueden dedicarse a su formación en tiempo parcial. Por eso su característica principal ha de ser la flexibilidad:

- En el tiempo, permitiendo que el usuario acceda a la educación cuando le sea posible.

- En el espacio, recibiéndola en el entorno más favorable.

- En el contenido, permitiendo, dentro de ciertos límites, la elección de las materias a estudiar en cantidad y contenido.

- En el ritmo de aprendizaje, dejando libertad al alumno, para adaptarlo a sus disponibilidades y a su capacidad.

El camino a seguir para conseguir esta flexibilidad está en una educación abierta y a distancia. Abierta significa flexibilidad y accesibilidad y a distancia es sinónimo de independencia y autonomía.

La enseñanza abierta incluye una serie de elementos de flexibilización que la hace más accesible a los estudiantes potenciales que los cursos tradicionales impartidos bajo la concepción actual. La mayoría de las veces, - por no decir siempre, - la apertura de la enseñanza se consigue con el auxilio de nuevos medios de información y comunicación.

La enseñanza a dstancia es una enseñanza que no se encuentra bajo la inmediata y continua supervisión del tutor, pero que se beneficia de la planificación, guía y control de la organización tutorial. Por su propia naturaleza goza de unas elevadas proporciones de independencia y autonomía.

Precisamente esta necesidad ha favorecido la implantación de centros públicos y privados de diferentes niveles que atienden con su organización a las necesidades de formación y reciclado de personas que no pueden asistir a los cursos regulares presenciales. 


\section{CONCLUSIONES}

Este trabajo ha constituído el inicio de una serie de cambios necesarios en la enseñanza de la Electrónica para los Ingenieros Técnicos Industriales para adecuarla a las condiciones actuales donde los egresados se insertan en ambientes de trabajo cada vez más competitivos y es deber de la universidad prepararlos para dar respuesta a las importantes transformaciones tecnológicas que se están produciendo.

Los resultados de esta investigación sugieren una revisión urgente de los modelos pedagógicos empleados y del sistema educativo en su generalidad para lograr en los estudiantes aprendizaje significativo y activo y poder brindar a los egresados una educación flexible en tiempo, espacio, contenidos y ritmos de aprendizaje.

\section{REFERENCIAS BIBLIOGRAFICAS}

1. AUSUBEL, D. The psicology of meaninful verbal learning. New York. Grune \& Straton. 1963.

2. BRUNER, J.J. Desarrollo cognitivo y educación. España. Morata. 1988

3. FERNANDEZ, M. et alt. El uso del ordenador en la enseñanza. Impacto de las nuevas tecnologías. Educación y nuevas tecnologías. Tendencias actuales. Febrero, 4. 1992, Madrid. p 30-35.

4. FERnANDEZ, M. et alt. Multimedia y Pedagogía. Un binomio actual. Memorias del Congreso Iberoamericano de Informática Educativa, vol.2, Junio 1992, Santo Domingo, p 333147.

5. IRDAC. Industrial Research and Development Advisory Committee of the Commission of the European Communities, España, Nov. 1990.

6. NIELSEN, J. Hypertext and Hipermedia. San Diego CA: Academic Press, 1990

7. SUMMERS, M. et alt. Improving primary school teachers understanding of science concepts - theory into practice, Journal Scienc. Educat, vol.14, (no.1)1990,. p 10-14

8. TABA, H. Teaching strategies and cognitive fuctioning. Cooperative Research Proyect, S. Francisco State College. 1966. 